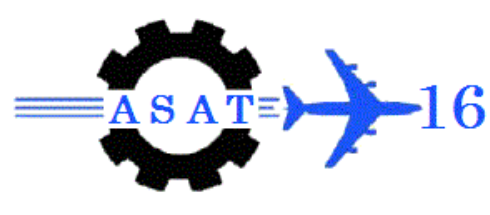

\title{
Compressed Watermarked Video Frame Transmission System Using Chaotic Interleaving
}

\author{
E. M. El-Bakary, S.EL-Rabaie, O. Zahran and F. E. Abd El-Samie
}

Department of Electronics and Electrical Communications Engineering, Faculty of Electronic Engineering, Menoufia University, 32952Menouf, EGYPT.

\section{E-mails:}

eman_elbakary449@yahoo.com, osama_zahran@menofia.edu.eg, srabie1@yahoo.com, and fathi_sayed@yahoo.com

\begin{abstract}
In this paper, we propose a chaotic Baker interleaving approach for efficient spiht compressed steganography watermarked frame of transmission of video over (OFDM) wireless systems with equalization. Cases of Rayleigh fading channels and additive white Gaussian noise are considered. The spiht compressed steganography watermarked video frames is primarily converted to approach of chrominance and after that the chrominance approach is converted to a binary data format. Prior to the modulation step made interleaving on the binary data. The proposed chaotic interleaving approach made reducing in the channel effects on the data which it is transmitted and made adding a degree of encryption to the transmitted data. The big data of the video frames is reduced by madding spiht compressed technique. To test the performance of the proposed approach by the transmission of spiht compressed steganography watermarked video various frames over additive white Gaussian noise and Rayleigh fading channels with interleaving by chaotic baker map. The results of the experiments explain that the received compressed steganography watermarked video frames are gives higher peak signal to noise ratios (PSNRs) with applied chaotic interleaving compared with other approaches and also show the histogram count of the correlation coefficient of the watermarked blocks.
\end{abstract}

Keywords: Video, Data Hiding and Compression, Steganography, DCT Compression, SPITH Video Compressed, OFDM and Chaotic Map.

\section{Introduction}

The most use of the video and steganography scheme is used in wide range of applications and is become interest, especially when steganography is used in cryptography with conjunction. Steganography technique is the technique that inserts the secret hiding data in the media .When we made combination of these techniques, the secret hiding data is authenticated embedded and encrypted in the target which we need it.The applications of steganography is split into usual application such as watermarking and the steganography technique is unusual applications such as video compression, which is our study. 
The process of steganography adds secret hiding data in the target which made for authentication and encryption, the compression process try to minimize the size of original file. The compression algorithms of the video frame is depends on DCT video frame. DCT is used for compression of video frames. We use stenographic scheme and the process of DCT compression together to decrease the size of the original file. The video frame is divided into a set of non-overlapping blocks and then some of these blocks are embedded into their subsequent blocks of the same video frame. So, the process of compression is made in two steps. The first step is used to perform DCT. The second step is made embedding some bitsnon overlapping blocks in its subsequent non overlapping blocks of the same video frame by algorithm of steganography. The algorithm of steganography cause decreasing in the size of file [1-8].

The process of embedding is depends on the projection, quantization and perturbation in the DCT domain bits of 8 blocks $\times 8$ block. Video frame is converted to luminance and chrominance components form. The chrominance component is embedded into the luminance component. The places of embedding are sub bands which the man eye can't see them. In our study, algorithm DCT Stego-video frame which gives high quality and high compression gain.

The objective of the SPHIT algorithm in this system is to decrease the amount of data transmitted to decrease the required bandwidth and the other objective is measure the performance enhancement of compressed watermarked video frames transmission systems through apply interleaving on the data. The transmission of video data through wireless channels face severe conditions such as burst errors which these errors are occur in clusters form. The effect of burst errors must be reduced so that it is applied interleaving on binary data of the compressed watermarked video frames [9-11].The performance of block interleaving techniques is limited [12-13]. To build strong interleavers it can be used chaotic maps scheme which based on chaotic Baker map which it is applied to the compressed watermarked video frames in binary format before the modulation step [14-20]. The advantages of chaotic maps randomization is minimize the channel effects without the need for coding schemes to detect the error and correct it. Another advantage of chaotic maps randomization is achieves a degree of encryption in the transmitted data which adds a security to the compressed watermarked video frame transmission process.Using linear equalization cause eliminating effects of the ISI and MAI [21-24].

Using the hybrid scheme randomization and equalization techniques improves the performance of OFDM systems. We apply the randomization on the spiht compressed watermarked video frames before transmission and apply the equalization step at the receiver. In the proposed scheme, it is required to estimate of the signal to noise ratio (SNR) to measure the efficiency of compressed watermarked video frames which it is received.

The organization of this paper is shown as follows. Section 2 explain the background on data hiding and compression. In Section 3, explained the suggested steganography watermarked scheme. In Section 4, explained the SPIHT Compression Algorithm .In Section 5 , explain the model of OFDM system which used for transmission of compressed watermarked video frame. Section 6 discusses the linear equalization. Section 7explain the chaotic Baker map randomization. Section 8 show the proposed model OFDM system with the hybrid scheme of chaotic interleaving randomization and equalization. Section 9 show the simulation results. At the end, Section 10 present remarks of the concluding.

\section{Background on compression and secret hiding data}

The goal of steganography is embedded data. Steganography has many applications. It is used in security techniques, government individuals, agencies, and through intellectual pursuit. The objectives of Steganography scheme are detectability, robustness and capacity of the hidden data, these advantages made Steganography is the most important than other 
techniques such as cryptography and watermarking. The background of the paper is presented in this section. Sections 2.1 explain the lossy DCT video compression scheme, while Section 2.2 presents some back ground on secret hiding data in video frames.

\subsection{Video}

Each video contain a number of frames, which need to be compressed via a transformation from the RGB color coordinate system to the Y CB CR system. Each component of the YCBCR system is divided into non overlapping blocks with size $8 \times 8$ pixels. The discrete cosine transform maps the pixels of $8 \times 8$ blocks into a set of transform coefficients [3]. The quantization and compression are performed for reducing the number of bits used to represent each coefficient. Quantization process is made on every coefficient. In a zigzag order, the coefficients are coded from low frequency coefficients to high frequency coefficients. By run-length coding the AC coefficients are coded. The video frame encodes the difference between the DC coefficients of consecutive blocks using a differential pulse code [4].

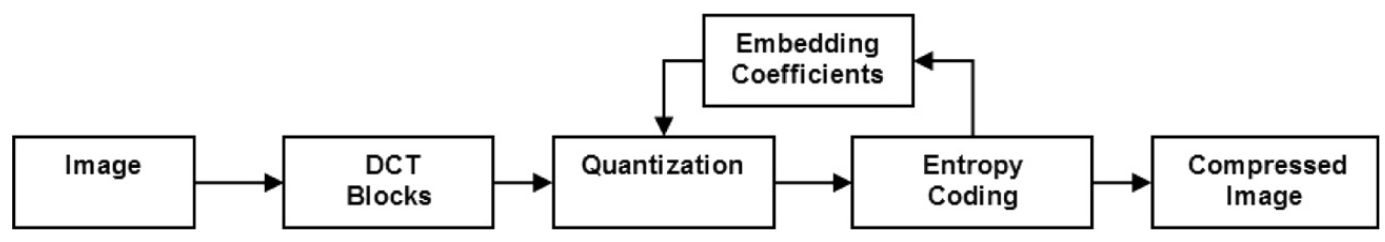

\section{DCT embedding process block diagram.}

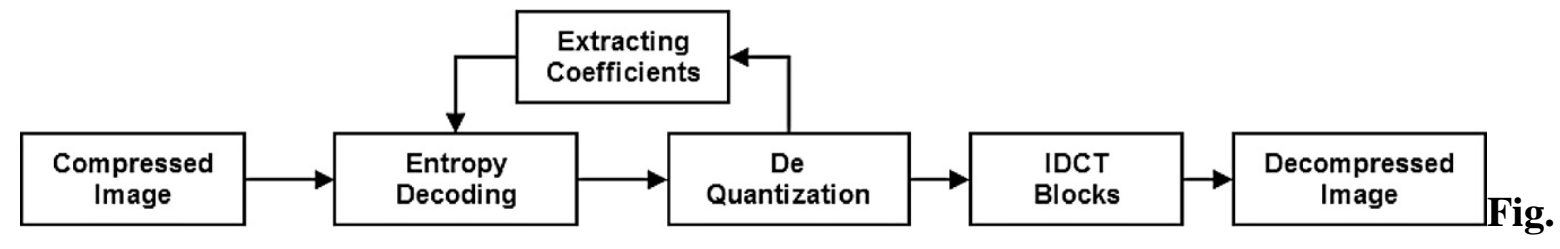

Fig.2. DCT extraction process block diagram.

\section{Hiding and compressed video scheme}

Steganography is the technique which made hiding secret objects in invisible manner. The advantages of steganography based compression algorithms are robustness, payload, invisibility, and the requirements of the size of the file. At The embedded process, the data which we embed it isn't visible so any one can't found it so it is called hiding data. The number of hidden bits represents the payload which determines the invisibility requirement. The Steganography achieve the robustness which embedded hiding data is detected after compression process. The file size after embedding is last requirement. The file size requirement is steganography application which the steganography cause reducing in the file size. The method which suggested in our work has two processes which are embedding processes and extraction processes [5].

\subsection{DCT-based scheme}

The target video frame is splitted into blocks which are none overlapping and the size of each block is $8 \times 8$ of 8 steganography. Target block is the blocks which call hidden block, and the blocks which are called subsequent are called the receiving blocks, which the target block is consider the source to give bits to the receiving blocks which this bits called receiving bits. We compress a target block by of video frame, and after that we hide the resulting bits into subsequent blocks $\mathrm{B}_{\mathrm{K}+1, \ldots \mathrm{B} 1}$ of the video frame which it is compressed. In video frames, each component $\mathrm{Y} \mathrm{C}_{\mathrm{B}} \mathrm{C}_{\mathrm{R}}$ respectively will be processed in separate manner. The all number of the

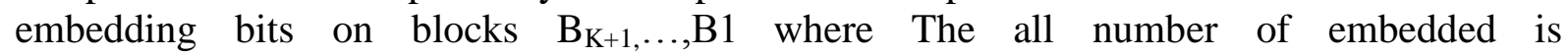
$\mathrm{n}=\mathrm{n}_{\mathrm{k}+1}+\mathrm{n}_{\mathrm{k}+2}+\ldots . .+\mathrm{n}_{1}$. The number of hidden bits per block is called $\mu$, so the size of the 
payload is equal to $\mu \times$ [ $\mathrm{N} \times \mathrm{M} / 8 \mathrm{X} 8]$. The data isn't hiding in the DC component, which the hiding data coefficients are embedded in high frequency coefficients.

After the embedded process which embedded bits, the value of quantized coefficient $C_{i}^{-}(i, j)$ of the blocks which called receiving blocks is rounded to the nearest even value or odd value. $C_{i}^{-}(i, j)$ is rounded to its odd value if the embedded bit is equal to $0, C_{i}^{-}(i, j)$ is rounded to its even value if the embedded bit isn't equal to 0 .

The performance of the embedding process is made in zigzag manner in the receiving blocks for preserving the quality of the frame. The embedding process is used threshold $\mathrm{T}$. If $\mathrm{T}$ increases, this causes that, for embedding little coefficients are used, so the size of the payload is minimized. In this study, the steganography scheme integrates embedding and rounding which increasing compression, so if $\mathrm{T}=0$ this cause maximizing embedding portion which the size of the file is increasing file size, and if $T=1$ this cause maximizing embedding portion.

The embedding process for each components $\mathrm{Y}, \mathrm{C}_{\mathrm{B}}$, and $\mathrm{C}_{\mathrm{R}}$ of video frame separately are summarized as follows:

\subsubsection{Algorithm1. Stego-JPEG video frame (DCT) encoder}

$\mathrm{I} / \mathrm{P}$ : video frames $\mathrm{I}$ of $\mathrm{N} * \mathrm{M}$ pixels is quantized to quantized coefficients of block $\mathrm{B}_{\mathrm{i}}$, where i $\varepsilon[1,(\mathrm{~N} x \mathrm{M}) /(8 \mathrm{x} 8)]$.

O/P: DCT Stego-video compression.

The embedding process are executed in the following steps:

1. rounding step is applied to block $B_{k}$

2. Compress $\mathrm{B}_{\mathrm{k}}$ by using JPEG.

3. The compressed block $B_{k}$ is hide the current bits in the $n$ coefficien subsequent blocks $\mathrm{B}_{\mathrm{k}+1}, \ldots, \mathrm{B}_{1}$ where

the number of coefficients $n=n_{k+1}+n_{k+2}+\ldots \ldots+n_{l}, n_{i}$ is used in the $i^{\text {th }}$ block such that

$$
C_{i}(i, j)=\left\{\begin{array}{cc}
Q_{b}\left(C_{i}^{-}(i, j)\right) & \left|C_{i}^{-}(i, j)\right| \geq T \\
{\left[C_{i}^{-}(i, j)\right]} & T-1 \leq\left|C_{i}^{-}(i, j)\right| \\
{\left[C_{i}^{-}(i, j)+0.5\right]} & \text { Otherwise }
\end{array}\right.
$$

\section{Repeat $1-3$ until $\mathrm{k}>[\mathrm{N} \times \mathrm{M} / \mathrm{m} \times \mathrm{m}], \mathrm{K}=l+1$.}

\subsubsection{The process of extraction}

The process of extracting is extract the embedded blocks from a compressed video frame explained as the following. The video frame is decoding for performing the decompression process. The extract of the embedded blocks are made by checking even $C_{i}(i, j)$ coefficients and odd $\mathrm{C}_{\mathrm{i}}(\mathrm{i}, \mathrm{j})$ coefficients from $\mathrm{n}$ coefficients of blocks which are call sequential blocks [7]. All coefficients are disregards by the decoder that quantize to a value with magnitude $\mathrm{T}$ or smaller. At the last, to extract blocks and original blocks, the inverse DCT is performed [8-9]. Repeated separately the process of DCT Stego decoder for every of the components $\mathrm{Y}, \mathrm{C}_{\mathrm{B}}$, and $C_{R}$ for video frame. The figure of decompression process is shown in Fig. 2.

\section{Algorithm2. Stego-JPEG video frame (DCT) decoder}

I/P: DCT Stego- video frame compressed.

$\mathrm{O} / \mathrm{P}$ : video frame decompression $l$.

The embedding process is executed in the next steps: 
1. Perform decoding process to decompress blocks.

2. Extract the embedded block of $B_{k+1}, \ldots, B_{1}$ coefficients $C_{i}(i, j) \geq T$ from the subsequent blocks which:

Embedded - bit $= \begin{cases}1 & \mathrm{Ci}(\mathrm{i}, \mathrm{j})=\text { odd } \\ 0 & \mathrm{Ci}(\mathrm{i}, \mathrm{j})=\text { even }\end{cases}$

3. For embedded block make decoding process.

4. The steps $1-3$ repeat it until $1>[\mathrm{N} * \mathrm{M} / \mathrm{m} * \mathrm{~m}], l=l+1$.

\section{Model of OFDM System}

OFDM is communication technology which are used in wireless systems of communications. High-speed data which transmitted using OFDM is converted from series data into parallel data of $\mathrm{N}$ sub channels $[1,2]$. So, apply modulation scheme such as binary phase shift keying on the data of each parallel sub channels which are transmitted. After the modulation, apply an inverse fast Fourier transform (IFFT) on the output of modulation data are to generate the multicarrier OFDM signal. Inserting guard intervals between frames to minimize the inter symbol interference (ISI) which cause by the multipath fading channels, [1,2]. The OFDM system block diagram is shown in Fig. (4).

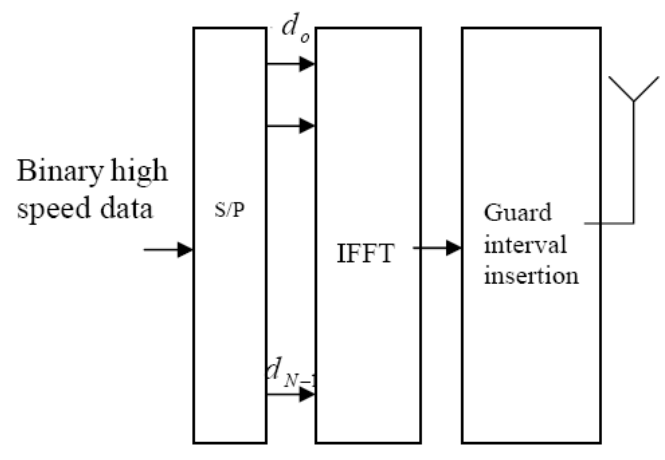

(a) Transmitter.

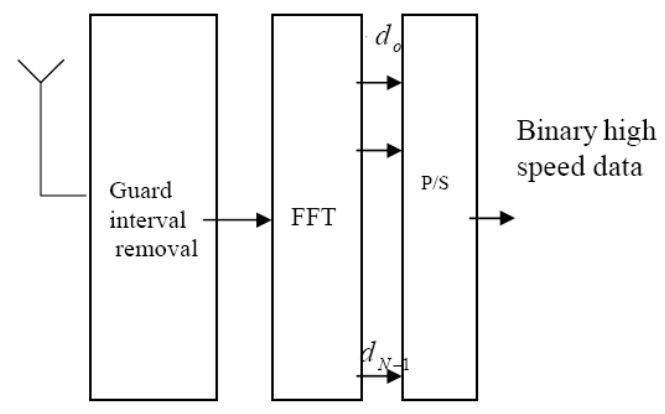

(b) Receiver.

Fig.(4) Model of OFDM System.

The form of guard intervals is added a cyclic prefix (CP) or zeros. The widely used approach is the addition of a CP. The OFDM use of the fast Fourier transform (FFT) and the inverse fast Fourier transform (IFFT), so that cause low complexity. Using the IFFT/FFT cause an ISI-free channel, a circular convolution is provide by the channel [1,2]., Figure (5) show Adding a CP to the transmitted signal, which provide the property of the circular convolution to the channel.

Adding a guard band of at least v samples between OFDM symbols this cause each OFDM symbol is independent to others OFDM symbols which coming before and after it. If we 
assume $\mathrm{T}_{\mathrm{s}}$ is the $\mathrm{T}_{\mathrm{g}}$ is the $\mathrm{CP}$ time, the symbol is $\mathrm{T}_{\text {total }}[1,2]$ :

$\mathrm{T}_{\text {total }} \quad=\mathrm{T}_{\mathrm{g}}+\mathrm{T}_{\mathrm{s}}$.

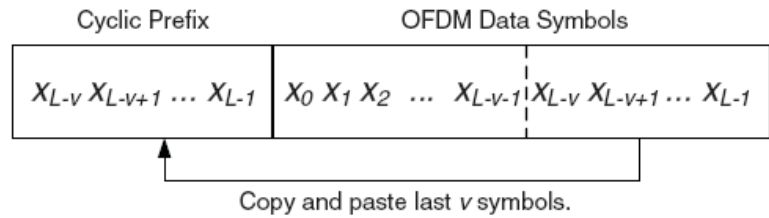

symbol time, and total duration of the

Fig. (5) Addition of the CP to an OFDM symbol.

The effect of the ISI can be minimized if the multipath delay or the channel impulse response is lower than the guard interval. This is occur however, the in-band fading or the inter carrier interference (ICI) is still found. The ratio between the usual symbol duration and the guard interval is dependent, as adding of a guard interval cause reducing the data throughput. The OFDM signal which transmitted is given by [14]:

$$
s^{\prime}(t)=\sum_{k=-\infty}^{\infty} \sum_{i=0}^{N-1} d(k) \exp \left(j 2 \pi f_{i}\left(t-k T_{\text {total }}\right)\right)
$$

where the data sequence is $\mathrm{d}(\mathrm{k})$ and the modified pulse waveform of each symbol is $f^{\prime}(t)$ is defined as[14]:

$$
f^{\prime}(t)= \begin{cases}1 & \left(-T_{g} \leq t \leq T_{s}\right) \\ 0 & \left(t<-T_{g}, t>T_{s}\right)\end{cases}
$$

The OFDM signal is transmitted through the wireless channel. A multipath fading channel and contaminated by additive white Gaussian noise (AWGN) is apply on the transmitted data $s^{\prime}(t)$. The received signal is defined as [14]:

$$
r(t)=\int_{0}^{\infty} h(\tau) s^{\prime}(t-\tau) d \tau+n(t)
$$

where,

the impulse response of the radio channel at time $\tau$ is $h(\tau)$, and the AWGN is $\mathrm{n}(\mathrm{t})$.

To avoid distorting the received signal apply a band pass filter on he received signal $r(t)$. After that removed the guard interval and apply an orthogonal detector to the signal to obtain the Fourier coefficients of the signal in the observation periods. $\hat{d}_{i}(k)$ is the FFT output circuit of the $i^{\text {th }}$ OFDM sub channel is given by [14]:

$$
\hat{d}_{i}(k)=1 / T_{s} \int_{k T_{\text {total }}}^{T_{s}+k T_{\text {otal }}} r(t) \exp \left(-j 2 \pi f_{i}\left(t-k T_{\text {total }}\right)\right) d t
$$

\section{Linear Equalization}

To eliminate the ISI which caused by the multipath fading channel and improvement the performance of the communication system is made by using linear equalization which is an efficient technique to made that. The linear equalization have different kinds in frequency domain such as the zero forcing (ZF) equalizer and the regularized zero forcing (RZF) equalizer and the linear minimum mean square (LMMSE) equalizer. The solution of ZF is defind as [21]:

$$
\mathbf{W}_{Z F}=\left(\mathbf{H}^{H} \mathbf{H}\right)^{-1} \mathbf{H}^{H}
$$

where the channel matrix is $\mathbf{H}$. The advantages of the frequency domain ZF is source data of the equalizer are not required and statistics of the additive noise aren't required too. The 
disadvantages of the frequency domain $\mathrm{ZF}$ equalizer are that, $\mathrm{ZF}$ equalizer causes noise enhancement and the computations needed for matrix inversion are time consuming. A new regularization part is added into Eq. (6) for the problem of noise enhancement in the ZF equalizer and become that [22-24]:

$$
\mathbf{W}_{R Z F}=\left(\mathbf{H}^{H} \mathbf{H}+\alpha \mathbf{I}\right)^{-1} \mathbf{H}^{H}
$$

Where, a parameter of regularization is $\alpha$. The resulting equalizer is called RZF equalizer. So in the RZF equalizer the additive noise and the statistics of the transmitted data are not required. This cause abetter equalizer which eliminate the mean square error (MSE) and remove the ISI. When $\alpha=1 / \mathrm{SNR}$, this achieve equalizer which is called the LMMSE equalizer. LMMSE equalizer is better than the $\mathrm{ZF}$ linear equalizer, as its better treatment to noise. The LMMSE is defined by [21]:

$$
\mathbf{W}_{\text {LMMSE }}=\left(\mathbf{H}^{H} \mathbf{H}+\frac{1}{S N R} \mathbf{I}\right)^{-1} \mathbf{H}^{H}
$$

\section{Chaotic Interleaving}

A chaotic map type which used to made interleaving is called Baker map which generates a square matrix in a permuted version. The Baker map is used to randomize a square matrix of data in its discretized form which represent an $M \times M$ matrix as follows [15-20]:

$$
B(r, s)=\left[\frac{M}{n_{i}}\left(r-M_{i}\right)+s \bmod \left(\frac{M}{n_{i}}\right), \frac{n_{i}}{M}\left(s-s \bmod \left(\frac{M}{n_{i}}\right)\right)+M_{i}\right]
$$

where the new indices of the data item are $B(r, s)$ at $(r, s), \mathrm{M}_{\mathrm{i}} \leq \mathrm{r}<\mathrm{M}_{\mathrm{i}}+\mathrm{n}_{\mathrm{i}}, 0<\mathrm{s}<\mathrm{M}$ and $\mathrm{M}_{\mathrm{i}}=\mathrm{n}_{1}+\mathrm{n}_{2}+\ldots+\mathrm{n}_{\mathrm{i}}$.

The perform of the chaotic interleaving steps explain as

1. Square matrix $M \times M$ is divided into $k$ vertical rectangles of width $n_{i}$ and height $M$.

2. Stretch vertical rectangles in the horizontal direction and after that made contracting vertically to obtain an $\mathrm{n}_{\mathrm{i}} \times \mathrm{M}$ horizontal rectangle.

3. Madding stack on horizontal rectangles as shown in Figure (6-a), where the right one is put at the top and the left one is put at the bottom.

4. Divided each output $n_{i} \times M$ vertical rectangle into $n_{i}$ boxes which its dimensions are $M=n_{i}$ $\times \mathrm{n}_{\mathrm{i}}$ and exactly containing $\mathrm{M}$ points.

5. Mapped each box column by column into a row as explain in Figure (6-b).

Figure 6 explain an example of chaotic interleaving of an $(8 \times 8)$ square matrix which use the secret key, $\mathrm{S}_{\mathrm{key}}=\left(\mathrm{n}_{1}, \mathrm{n}_{2}, \mathrm{n}_{3}\right)$

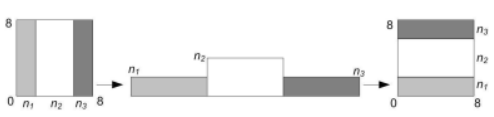

$=(2,4,2)$.

(a)

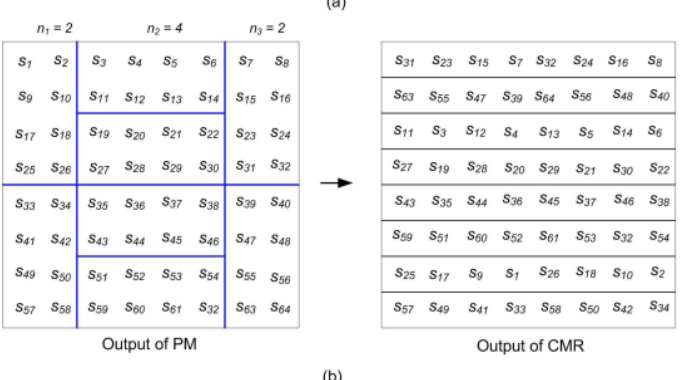

Fig. (6) Interleaving by Chaotic. (a) Baker map in discretize form. (b) A $8 \times 8$ randomization matrix.

\section{The Proposed Model of Chaotic Map with OFDM and Equalization System}

There are 4 stages in this proposed model to transmit spiht compressed steganography watermarked video frames .The first stage is spiht compressed steganography watermarked frame of video data formatting, the second stage is a chaotic interleaving on the binary video data, the third stage is OFDM modulation and the final stage is equalization. The proposed 
system model block diagram is explain in the following Fig. (7). The proposed model OFDM system steps explain as follows:

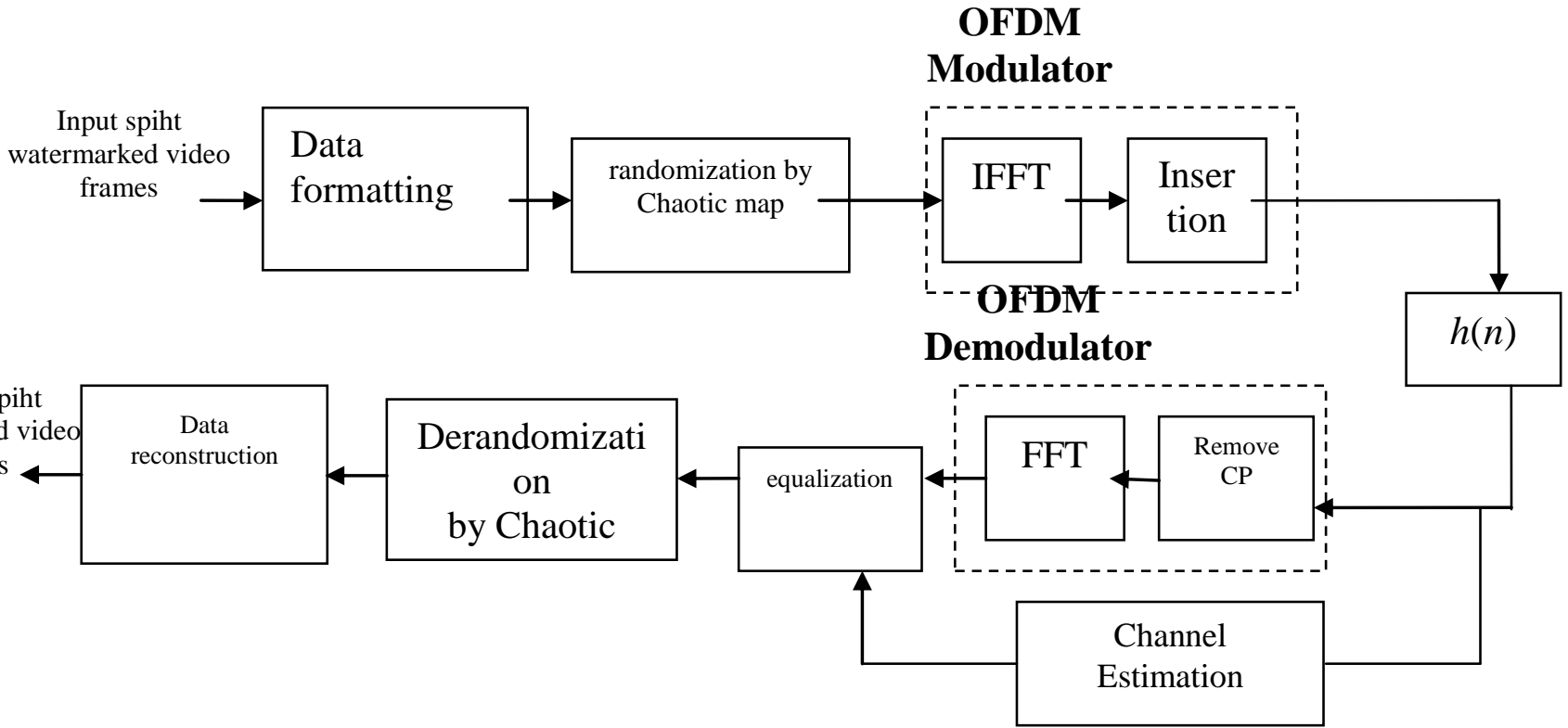

Fig. (7) The chaotic interleaver and equalization with OFDM block diagram.

1. The spiht compressed steganography watermarked video frame is converted to the binary matrix form.

2. Reshape the binary matrix which is non-square to an $\mathrm{M} \times \mathrm{M}$ square binary matrix format.

3. Apply baker chaotic map randomization to square binary matrix format.

4. Reshape the square binary matrix to its original dimensions.

5. Perform the OFDM modulation on the binary data.

6. Apply the OFDM demodulation at the receiver this process is reversed to obtain channel estimation, assume that the receiver has the secret key of the chaotic map which the same secret key at the transmitter. After that, the received signal is equalized to suppress the ISI, equalizer is called the LMMSE equalizer as follows:

$$
\hat{\mathbf{d}}=\left(\mathbf{H}_{d}^{H} \mathbf{H}_{d}+\alpha \mathbf{I}\right)^{-1} \mathbf{H}_{d}^{H} \mathbf{r}
$$

7. Reshape binary data to a square matrix and apply achaotic map derandomization to binary matrix.

8. Reshape the square binary matrix to its original dimensions.

9. Retrieve the spiht compressed steganography watermarked video frame from the binary data.

\section{Results of Simulation}

In our study we use the DCT Stego-JPEG scheme video frame compression. The performance of the compression scheme under consideration is determined by using correlation coefficient between blocks and length vector containing histogram counts for correlation coefficient of blocks which counts the number of values in correlation coefficient of blocks that fall between the elements in the correlation coefficient vector (which must contain monotonically non-decreasing values).Comparison is the resemblance between correlation coefficient rang and length vector containing histogram counts for correlation coefficient of blocks for video frame. For the comparison between video frames, we employed histogram counts for correlation coefficient of blocks as the measure of video frame quality. Our results show that 
increasing number of values in correlation coefficient of blocks that fall between the elements in the correlation coefficient vector which is increasing with acceptable quality for DCT Stenography JPEG as shown in Fig. 8. To measure the quality of the reconstructed spiht compressed steganography watermarked video frames at the receiver use PSNR the peak signal to noise ratio. PSNR is the ratio between the maximum power of a signal and the power of corrupting noise. PSNR is usually expressed in decibel scale. To achieve accuracy by using comparing between the various steganography watermarked video frames. The MSE is squared error between the compressed and the original steganography watermarked video frames, where PSNR is the measurement of the peak error. The MSE and PSNR are explain as follows.

$$
\begin{aligned}
& M S E=\frac{1}{M N} \sum_{y=1}^{M} \sum_{x=1}^{N}\left[I(x, y)-I^{\prime}(x, y)\right]^{2} \\
& P S N R=20 * \log \left(\frac{255}{\sqrt{M S E}}\right)
\end{aligned}
$$

where the original steganography watermarked video frames is $\mathrm{I}(\mathrm{x}, \mathrm{y})$, the decompressed steganography watermarked video frames is $\mathrm{I}^{\prime}(\mathrm{x}, \mathrm{y})$ and the dimensions of the steganography watermarked video frames are M,N. To evaluate the performance and efficiency of the proposed scheme system, various monochrome video frames are used for more accuracy such as sat.avi video $256 * 256 * 3 * 922$ having $256 * 256 * 3$ pixels. These video frames are used as input to the simulation framework. So we use a chaotic map of size of $256 \times 256$ pixel for video frame of size $256 \times 256$ and we use a chaotic map of size of $256 \times 256$ pixel for video frames of size $256 \times 256$.

To test the performance of the proposed scheme carried out many simulation experiments to achieve accuracy. The model of wireless channel which is used is The SUI3 channel where The SUI3 channel is one model of six channel models adopted by the IEEE 802.16a standard for evaluating the performance of broadband wireless systems in the $2-11 \mathrm{GHz}$ band [29]. SUI3 channel has three Rayleigh fading taps at delays of $0,0.5$ and $1 \mathrm{~ms}$ and relative powers of $0 \mathrm{~dB},-5 \mathrm{~dB}$, and $-10 \mathrm{~dB}$, respectively. The simulation parameters in table (1).

\begin{tabular}{|l||l|l|}
\hline \multirow{4}{*}{ Transmitter } & Modulation & BPSK \\
\cline { 2 - 3 } & video size & $256 \times 256 \times 3 \times 921$ \\
\cline { 2 - 3 } & Cyclic prefix & 20 samples. \\
\cline { 2 - 3 } & Transmitter IFFT size & M=256 symbols. \\
\cline { 2 - 3 } Chaotic map size & $128 \times 128$ \\
\hline \multirow{2}{*}{ Receiver } & Fading & SUI3 channel \\
\hline Environment of noise & AWGN \\
\hline & Equalization & LMMSE \\
\hline
\end{tabular}

\section{Table (1) Simulation Parameters.}

In the first experiment, In sat.avi video, SPIHT compressed steganography watermarked video frame is transmitted using OFDM with only equalization and OFDM with equalization and with chaotic interleaving. OFDM system model with only equalization at $\mathrm{SNR}=10 \mathrm{~dB}$ for frame 900 from sat.avi video, the PSNR will be $17.79 \mathrm{~dB}$, this value is increased using equalization and randomization of video frame by the chaotic map OFDM system to be 18.22 $\mathrm{dB}$ as shown in Fig. (10) respectively. The chaotic map for this case reduces the noise. 
For $\mathrm{SNR}=30 \mathrm{~dB}$ for frame 900 from sat.avi video, the PSNR will be $25.11 \mathrm{~dB}$, this value is increased using equalization and randomization of video frame by the chaotic map to be 29.37dB as shown in Fig.(9) respectively. For $S N R=15 d B$, for frame 400 from sat.avi video the PSNR will be $15.10 \mathrm{~dB}$, this value is increased using equalization and randomization of video frame by the chaotic map to be $19.49 \mathrm{~dB}$ as shown in Fig.(12) respectively .

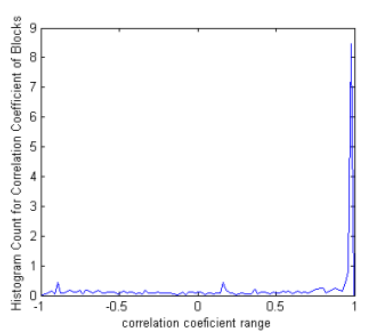

(a) The correlation coefficient between blocks

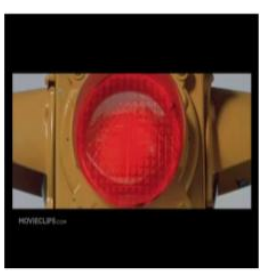

(b) Original steganography watermarked Frame 900

Fig (8) . Original steganography watermarked Frame 900 and the correlation coefficient between

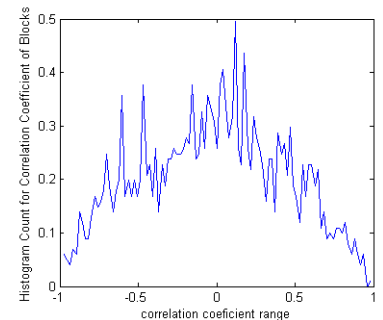

(a) The correlation coefficient between blocks in the case of using equalization and without randomization

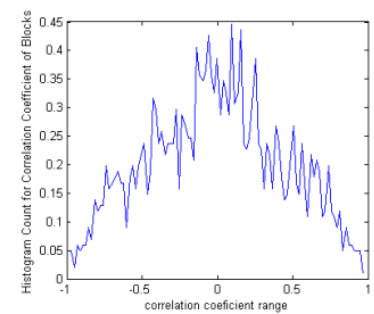

(c) The correlation coefficient between blocks in the case of using equalization and with randomization.

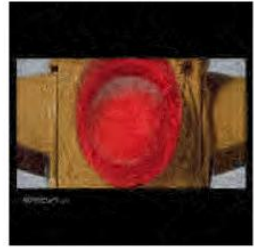

(b) $\mathrm{SNR}=30 \mathrm{~dB}$ spiht compressed steganography watermarked Frame $900 \mathrm{PSNR}=29.37 \mathrm{~dB}$ with randomization

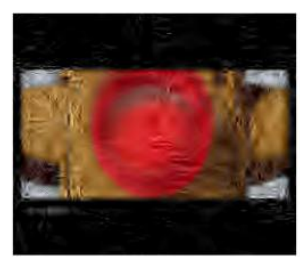

(d) $\mathrm{SNR}=30 \mathrm{~dB}$ spiht compressed steganography watermarked Frame $900 \mathrm{PSNR}=25.11 \mathrm{~dB}$ without randomization

Fig (9).The received Compressed SPIHT compressed steganography watermarked Frame 900 and the correlation coefficient between blocks using OFDM in the cases. SUI-3 Raleigh fading channel influenced by AWGN is used. $\mathrm{SNR}=30 \mathrm{~dB}$. 


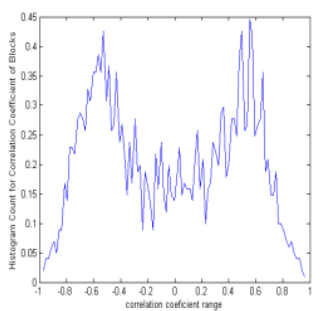

(a) The correlation coefficient between blocks in the case of using equalization and without randomization

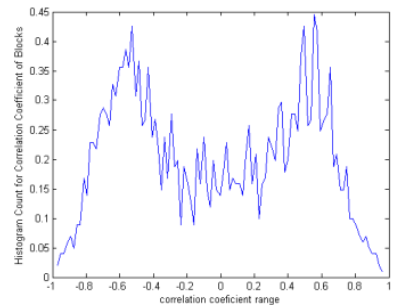

(c) The correlation coefficient between blocks in the case of using equalization and with randomization.

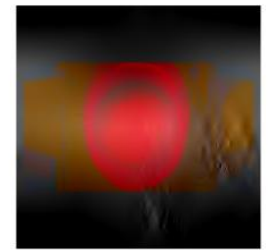

(b) SNR=10 dB SPIHT compressed steganography watermarked Frame 900 with equalization and randomization. PSNR $=18.22 \mathrm{~dB}$

Fig (10). The received Compressed SPIHT compressed steganography watermarked Frame 900 and the correlation coefficient between blocks using OFDM in the cases. SUI-3 Raleigh fading channel influenced by AWGN is used. $\mathrm{SNR}=10 \mathrm{~dB}$.

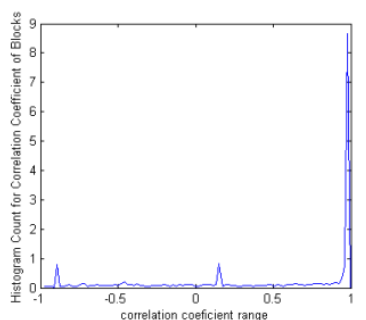

(a) The correlation coefficient between blocks

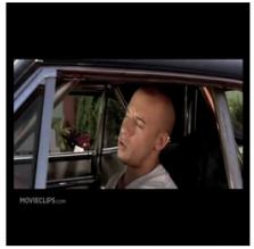

(b) Original steganography watermarked Frame 400

Fig (11). Original steganography watermarked Frame 400 and the correlation coefficient between blocks.

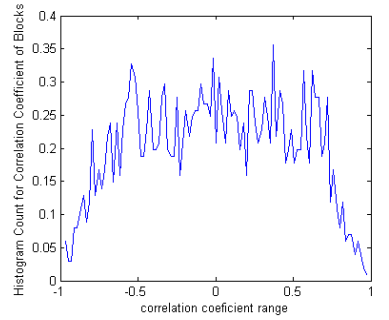

(a) The correlation coefficient between blocks in the case of using equalization and without randomization

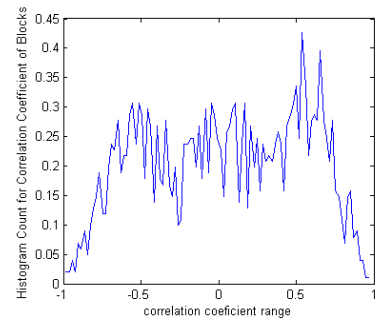

(c) The correlation coefficient between blocks in the case of using equalization and with randomization.

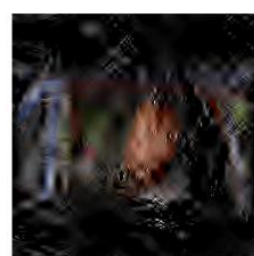

(b) $\mathrm{SNR}=15 \mathrm{~dB}$ SPIHT compressed steganography watermarked Frame 400 PSNR= $19.49 \mathrm{~dB}$ with randomization

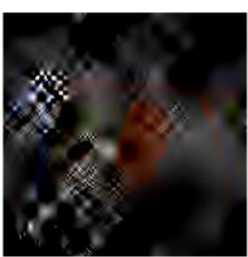

(d) SNR =15 SPIHT compressed steganography watermarked Frame $400 \mathrm{PSNR}=15.10 \mathrm{~dB}$ without randomization

Fig (12). The received Compressed SPIHT compressed steganography watermarked Frame 400 and the correlation coefficient between blocks using OFDM in the cases. SUI-3 Raleigh fading channel influenced by

AWGN is used. $\mathrm{SNR}=15 \mathrm{~dB}$. 


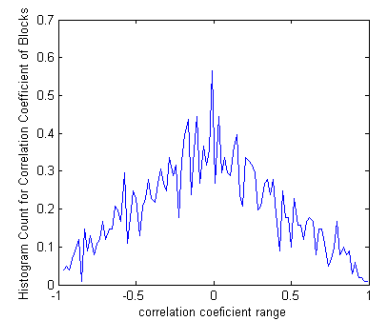

(a) The correlation coefficient between blocks in the case of using equalization and without randomization

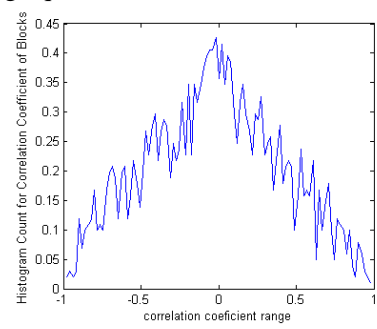

(c) The correlation coefficient between blocks in the case of using equalization and with randomization.

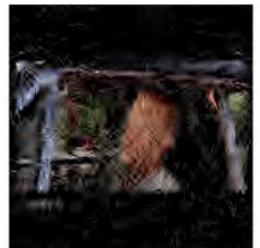

(b) $\mathrm{SNR}=30$ SPIHT compressed steganography watermarked Frame $400 \mathrm{PSNR}=22.51 \mathrm{~dB}$ without randomization

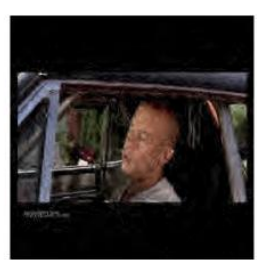

(d) $\mathrm{SNR}=30 \mathrm{PSNR}=29.58 \mathrm{~dB}$ SPIHT compressed steganography watermarked Frame 400 with randomization

Fig(13). The received Compressed SPIHT compressed steganography watermarked Frame 400 and the correlation coefficient between blocks using OFDM in the cases. SUI-3 Raleigh fading channel influenced by AWGN is used. $\mathrm{SNR}=30 \mathrm{~dB}$.

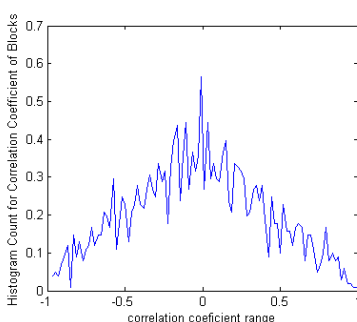

(a) The correlation coefficient between blocks in the case of using equalization and without randomization

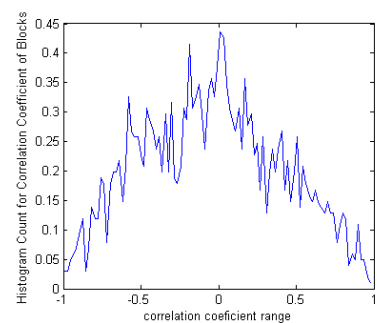

(c) The correlation coefficient between blocks in the case of using equalization and with randomization.

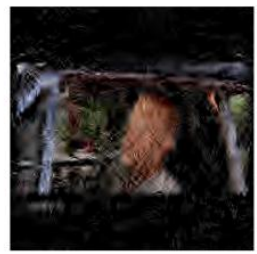

(b) $\mathrm{SNR}=50$ SPIHT compressed steganography watermarked Frame $400 \mathrm{PSNR}=22.51 \mathrm{~dB}$ without randomization

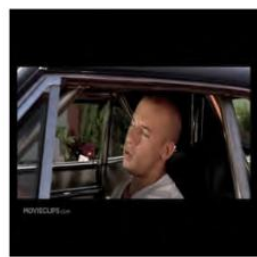

(d) $\mathrm{SNR}=50 \mathrm{PSNR}=38.64 \mathrm{~dB}$ SPIHT compressed steganography watermarked Frame 400 with randomization

Fig .(14). The received Compressed SPIHT compressed steganography watermarked Frame 400 and the correlation coefficient between blocks using OFDM in the cases. SUI-3 Raleigh fading channel influenced by

\section{Conclusion}

AWGN is used. $\mathrm{SNR}=50 \mathrm{~dB}$.

This paper has presented an efficient compressed steganography watermarked video transmission system through wireless channel using randomization technique (chaotic map) that applied on compressed watermarked video frame with linear equalization in OFDM for noise reduction. The use of chaotic map with linear equalization in OFDM serves additional purposes such as reducing ISI caused by multi-path fading channels and increases the PSNR. Results of the simulation depended on digital video frames which show a good match between objective performance metrics and human visual perception. This study gives a good 
results when applied chaotic map with linear equalization and OFDM at AWGN and Rayleigh fading channels.

\section{Reference}

[1] J. G. Andrews, A. Ghosh and R. Muhamed, Fundamentals of WiMAX, Pearson Education, Inc. 2007.

[2] W. Webb, Wireless Communications: The Future, John Wiley \& Sons Ltd, 2007.

[3] M. Khedr, M. Sharkas, A. Almaghrabi and O. Abdelaleem, " A SPIHT/OFDM with Diversity Technique for Efficient Image Transmission over Fading Channels", Proceedings of the IEEE International Coference on Wireless Communications, Networking and Mobile Computing (Wicom), pp. 480-483, 2007.

[4] J. fang, M. H. Lee, M. Jiang and J Y. Park, " Robust Wireless Image Transmission Based on Turbo-Coded OFDM", IEEE Transactions on Consumer Electronics, Vol. 48, No. 3, pp. 724-731, 2002.

[5] H. S. Chu, B. S. Park, C. K. An, J. S. Kang; H. G Son, " Wireless image transmission based on adaptive OFDM system", Proceedings of the IEEE International Forum on Strategic Technology, IFOST, pp.623-626, 2007.

[6] M.M. Salah, A. A. Elrahman, and M. M. Mokhtar, "Performance Enhancement of Image Transmission Using UEP Overlayed UTG_OFDM Scheme", Proceedings of the 25th National Radio Science Conference (NRSC), 2008.

[7] M. Farshchian and W. A. Pearlman, " Real-Time Video Transmission Over MIMO OFDM Channels Using Space-Time Block Codes", Proceedings of the IEEE $40^{\text {th }}$ Annual Conference on Information Sciences and Systems, pp. 1140-1145, 2006.

[8] T. Athanasiadis, K. H. Lin, and Z. M. Hussain, " Multimedia Transmission over Wireless Space-Time-Frequency coded OFDM Systems with Adaptive Beamforming", Proceedings of the IEEE Asia-Pacific Conference on Communications (APCC), 2006.

[9] Y.Wang and Q. F.Zhu, "Error control and concealment for video communication: A review, " Proceedings of the IEEE,Vol.86,pp.974-997, 1998.

[10] C. K. Sung, J. Heo, and I. Lee, "Adaptive Bit-Interleaved Coded OFDM with Reduced Feedback Information", IEEE Transactions on Communications, Vol. 55, No. 9, pp. 164911655, 2007.

[11] S.W. Lei and V. K. N. Lau "Performance Analysis of Adaptive Interleaving for OFDM Systems", IEEE Transactions On Vehicular Technology, Vol. 51, No. 3, pp. 435-444, 2002.

[12] Y. Fang, L. Yu, " Block-Interleaved Error-Resilient Entropy Coding", Proceedings of IEEE International Symposium on Circuits and Systems (ISCAS), pp.53-56 2007.

[13] V. D. Nguyen, H. P. Kuchenbecker, " Block interleaving for soft decisioln Viterbi decoding in OFDM systems", Proceedings of the IEEE Vehicular Technology Conference(VTC), pp. 470-474, 2001.

[14] H. Harada and R. Prasad, Simulation and Software Radio for Mobile Communications, Artech House Publishers, 2002.

[15] F. Han, X. Yu, . and S. Han, " Improved Baker Map for Image Encryption", Proceedings of the First International Symposium on Systems and Control In Aerospace and Astronautics, ISSCAA 2006, pp.1273-1276.

[16] J. Fridrich, "Image Encryption Based on Chaotic Maps", Proceedings of the IEEE International Conference on Systems, Man, and Cybernetics, 1997, pp. 1105-1110.

[17] Q. Qian , Z. Chen, Z. Yuan, "Video Compression and Encryption Based-on Multiple Chaotic

System", Proceedings of The 3rd International Conference on Innovative Computing Information and Control (ICICIC'08).

[18] F. Huang, F. Lei, "A Novel Symmetric Image Encryption Approach Based on a New Invertible Two-dimensional Map", Proceedings of the International Conference on Intelligent Information Hiding and Multimedia Signal Processing IIHMSP 2008, pp. 1340-1343. 
[19] S. C. Koduru and V. Chandrasekaran, "Integrated Confusion-Diffusion Mechanisms for Chaos Based Image Encryption", IEEE 8th International Conference on Computer and Information Technology Workshops, 2008, pp. 260-263.

[20] K. Usman, H. Juzojil and I. Nakajimal, "Medical Image Encryption Based on Pixel Arrangement and Random Permutation for Transmission Security" Proceedings of the $9^{\text {th }}$ International Conference on e-Health Networking, Application and Services, 2007, pp. 244247.

[21] A. Klein, "Data detection algorithms specially designed for the downlink of CDMA mobile radio systems," in proc. IEEE VTC, vol. 1, pp. 203-207, 4-7 May 1997.

[22] F. S. Al-kamali, M. I. Dessouky, B. M. Sallam, and F. E. El-Samie, "Frequency

Domain interference cancellation for single carrier cyclic prefix CDMA systems,"

Progress In Electromagnetics Research B, PIERB 3, pp. 255-269, 2008.

[23] F. S. Al-kamali, M. I. Dessouky, B. M. Sallam, and F. E. El-Samie, "Performance Evaluation of Cyclic Prefix CDMA Systems with Frequency Domain Interference Cancellation," Digital Signal Processing Journal, Elsevier Inc, Volume 19, Issue 1, pp. 2-13, January 2009.

[24] A. Klein, "Data Detection Algorithms Specially Designed for The Downlink of CDMA Mobile Radio Systems," in proc. of IEEE VTC, vol. 1, pp. 203-207, 4-7 May 